\title{
Shot noise of series quantum point contacts intercalating chaotic cavities
}

\author{
S. Oberholzer, ${ }^{1, *}$ E. V. Sukhorukov, ${ }^{2}$ C. Strunk, ${ }^{3}$ and C. Schönenberger ${ }^{1}$ \\ ${ }^{1}$ University of Basel, Klingelbergstr. 82, 4056 Basel, Switzerland \\ ${ }^{2}$ University of Geneva, 24, quai Ernest Ansermet, 1211 Geneve 4, Switzerland \\ ${ }^{3}$ University of Regensburg, D-93040 Regensburg, Germany
}

(Received 11 June 2002; published 6 December 2002)

\begin{abstract}
Shot noise of series quantum point contacts forming a sequence of cavities in a two-dimensional electron gas are studied theoretically and experimentally. Noise in such a structure originates from local scattering at the point contacts as well as from chaotic motion of the electrons in the cavities. We found that the measured shot noise is in reasonable agreement with our theoretical prediction taking the cavity noise into account.
\end{abstract}

DOI: 10.1103/PhysRevB.66.233304

PACS number(s): 73.23.Ad, 72.70.+m, 73.50.Td

\section{INTRODUCTION}

Shot noise is a nonequilibrium type of electrical current noise directly resulting from random transfer of discrete charge quanta. ${ }^{1}$ For Poissonian transfer of single electrons the spectral density of the current fluctuations is $S_{\text {Poisson }}$ $=2 e|I|$. Correlations imposed by Fermionic statistics or Coulomb interaction may change shot noise from $S_{\text {Poisson }}$ which is expressed by the Fano factor $F$ defined as $F$ $=S / S_{\text {Poisson }}$. This is, for example, the case for a one mode quantum wire with an intermediate barrier of transmission probability $T$ where the shot noise is suppressed by a Fano factor $F=1-T$ below its full Poissonian value. ${ }^{2,3}$ In various mesoscopic systems universal Fano factors have been found such as $F=1 / 3$ in metallic diffusive wires ${ }^{4,5}$ or $F=1 / 4$ in chaotic cavities. $^{6-8}$

In this paper the shot noise of a series of quantum point contacts forming a sequence of cavities in a two-dimensional electron gas is studied theoretically and experimentally. The noise in such a structure originates from local scattering at the point contacts as well as from diffraction of the electronic wave function within the cavities. While the Fano factor $F$ of a single scatterer equals $1-T$, with $T$ its transmission probability, $F$ reaches $1 / 3$ in the limiting case of an infinite number of scatterers. ${ }^{9}$ Thus the case of a large number of point contacts in series models a diffusive wire with randomly placed impurities, for which the Fano factor is also $1 / 3$. $^{4,5}$ The work presented here is devoted to this crossover from $F=1-T$ for a single scatterer to $F=1 / 3$ in the diffusive regime. Experimentally, this can be investigated by measuring shot noise of several quantum point contacts (QPC's) in series, which model the impurity scattering in a diffusive wire.

\section{CROSSOVER FROM A SINGLE SCATTERER TO THE DIFFUSIVE REGIME}

The shot noise of a sequence of $N$ planar tunnel barriers has been calculated by de Jong and Beenakker within a semiclassical description based on the Boltzmann-Langevin approach. ${ }^{9}$ For equal transmission probabilities $T_{i=1, \ldots, N}$ $=T$ the Fano factor is found to be

$$
F=\frac{1}{3}\left(1+\frac{N(1-T)^{2}(2+T)-T^{3}}{[T+N(1-T)]^{3}}\right) .
$$

According to this result $F$ indeed reaches $1 / 3$ with increasing barrier number $(N \rightarrow \infty)$ for any value of the transparency $T \in[0,1] .{ }^{10}$ In this description a diffusive conductor can be modeled as the continuum limit of a series of tunnel barriers. However, the calculation in Ref. 9 is only valid for onedimensional transport since it neglects transverse motion of the electrons in between the barriers. ${ }^{11}$ It is therefore not appropriate to describe our physical system consisting of a series of quantum point contacts (Fig. 1). Here, we consider the case that there are cavities between the barriers in which the electrons scatter chaotically leading to additional cavity noise. . $^{6,16}$

In general, the fluctuations in the total current through the system shown in Fig. 1 can be written as ${ }^{7,16}$

$$
\delta I=\delta I_{n}^{S}+G_{n}\left(\delta V_{n-1}-\delta V_{n}\right), \quad n=1, \ldots, N
$$

using the fact that the total current is conserved. $G_{n}$ $=G_{0} \Sigma_{k} T_{k n}\left(G_{0} \equiv 2 e^{2} / h\right)$ is the conductance of the $n$th QPC and $\delta V_{n}$ the voltage fluctuations inside the $n$th cavity. $\delta I_{n}^{S}$ are the current fluctuations of a single QPC:

$$
\left\langle\delta I_{n}^{S} \delta I_{m}^{S}\right\rangle=S_{n} \delta_{n m}
$$

with $^{2}$

$$
\begin{aligned}
S_{n}= & 2 G_{0} \sum_{k} \int_{0}^{\infty} d E\left[T_{k n} f_{n-1}\left(1-f_{n-1}\right)+T_{k n} f_{n}\left(1-f_{n}\right)\right. \\
& \left.+T_{k n}\left(1-T_{k n}\right)\left(f_{n-1}-f_{n}\right)^{2}\right] .
\end{aligned}
$$



FIG. 1. Schematics of the considered system: $N$ quantum point contacts forming a series of cavities. $f_{n=0, \ldots, N}$ denote the distribution functions of the electrons. In such a system shot noise arises due to quantum diffraction inside the cavity as well as to partitioning at the contacts. 
Summing the square of Eq. (2) over $n$, while assuming equal conductances $G_{n}=G$, the total noise power follows as

$$
S \equiv\left\langle\delta I^{2}\right\rangle=\frac{1}{N^{2}} \sum_{n, m=1}^{N}\left\langle\delta I_{n}^{S} \delta I_{m}^{S}\right\rangle=\frac{1}{N^{2}} \sum_{n=1}^{N} S_{n},
$$

where we have assumed $\delta V_{0}=\delta V_{N}=0$, i.e., no fluctuations in the potential of the perfect metallic leads.

\section{A. Noninteracting electrons}

The distribution function $f_{n}$ of the $n$th cavity follows from the conservation of numbers of electrons in each energy interval. ${ }^{7}$ For equal conductances of the $N$ point contacts $f_{n}$ is given by

$$
f_{n}(E)=\left(\frac{N-n}{N}\right) f_{L}(E)+\frac{n}{N} f_{R}(E),
$$

with $f_{L}(E)=f_{F}\left(E, e V_{L}, \theta\right)$ and $f_{R}(E)=f_{F}\left(E, e V_{R}, \theta\right)$ the equilibrium Fermi function in the left and right reservoir, respectively. $\theta$ denotes the bath temperature. For simplicity only one propagating mode will be considered for the moment with the backscattering parameter $\mathcal{R}_{n} \equiv 1-T_{n}=\mathcal{R}$ assumed to be the same for all barriers. Substituting the distribution function $f_{n}$ from Eq. (6) into Eq. (4), the total noise of $N$ point contacts in series follows from Eq. (5):

$$
\begin{aligned}
S= & \frac{4 G_{S} k_{B} \theta}{N^{2}}\left\{\frac{1}{3}\left[\left(2 N^{2}+1\right)+\left(N^{2}-1\right) \frac{e V}{2 k_{B} \theta} \operatorname{coth}\left(\frac{e V}{2 k_{B} \theta}\right)\right]\right. \\
& \left.+\mathcal{R}\left[\frac{e V}{2 k_{B} \theta} \operatorname{coth}\left(\frac{e V}{2 k_{B} \theta}\right)-1\right]\right\} .
\end{aligned}
$$

$G_{S}=G / N$ is the total conductance of the device. In the zerotemperature limit we obtain for the Fano factor

$$
F \equiv \frac{S}{2 e|I|}=\left(\frac{1}{3}-\frac{1}{3 N^{2}}+\frac{\mathcal{R}}{N^{2}}\right) .
$$

With this expression for the Fano factor $F$, Eq. (7) can be rewritten in a more simple and transparent form:

$$
\begin{aligned}
S= & S_{e q}\left[\frac{2}{3}+\frac{1}{3}-\left(\frac{1}{3}-\frac{1}{3 N^{2}}+\mathcal{R} N^{2}\right)\right. \\
& \left.+\left(\frac{1}{3}-\frac{1}{3 N^{2}}+\frac{\mathcal{R}}{N^{2}}\right)(\beta \operatorname{coth} \beta)\right] \\
= & S_{e q}[1+F(\beta \operatorname{coth} \beta-1)],
\end{aligned}
$$

with $S_{e q}=4 G_{S} k_{B} \theta$ and $\beta \equiv\left(e V / 2 k_{B} \theta\right)$.

For a single QPC $(N=1) F$ equals the backscattering parameter $\mathcal{R}=1-T$ as expected. For $N=2$ a single cavity is separated from the leads by two QPC's and for ideal contacts (i.e., $\mathcal{R}=0$ ) the Fano factor is $1 / 4$. If the QPC's are in the tunneling regime $(\mathcal{R} \simeq 1)$ the noise is dominated by the QPC's and the dynamics inside the cavity play no role. The Poissonian voltage noise of the two contacts adds up resulting in a Fano factor 1/2. In the intermediate regime $F$

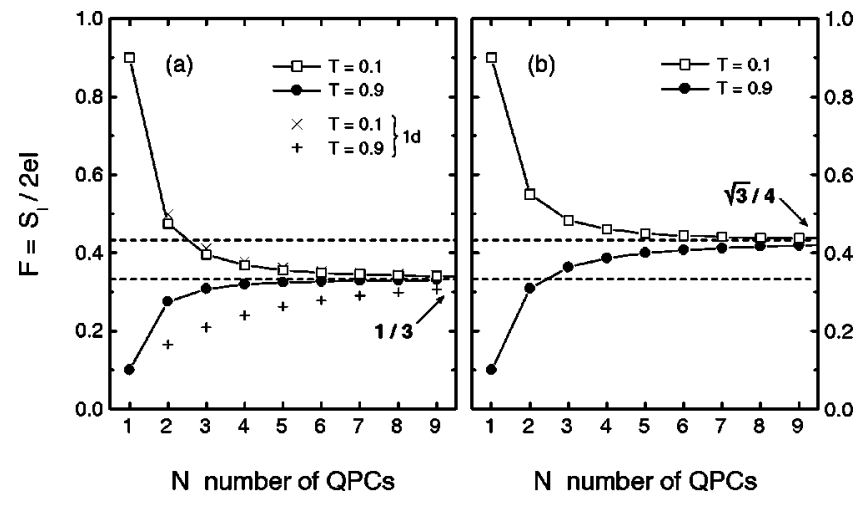

FIG. 2. Theoretical prediction for shot noise normalized to the Poissonian limit $2 e|I|$ as a function of the number of QPC's $N$ in series for cold (a) and hot electrons (b) at zero bath temperature. The crosses $(X,+)$ in the left plot correspond to a calculation of de Jong and Beenakker ${ }^{9}$ for one-dimensional transport, not including the noise arising from chaotic motion of the carriers in the cavities between the barriers. This is taken into account for Eq. (8).

$=\frac{1}{4}(1+\mathcal{R})$. Increasing the number of QPC's $(N \rightarrow \infty)$ the Fano factor $F$ reaches $1 / 3$ independent of $\mathcal{R}=1-T$ as for the calculation in Ref. 9.

In Fig. 2(a) the result of Eq. (8) is compared to the result for one-dimensional tunnel-barriers. ${ }^{9}$ For point contacts with low transparencies $(T=0.1)$ the results are very similar because in this case the noise is dominated by the contacts. But for high transparencies $(T=0.9)$ the Fano factor including "cavity noise" [Eq. (8)] increases much faster with the number of QPC's than the one-dimensional model of Ref. 9.

\section{B. Interacting electrons}

In case of electron-electron interaction within the cavities the distribution function inside the $n$th cavity $f_{n}(E, \theta)$ equals a Fermi function $f_{F}\left(e V_{n}, \theta_{n}\right)$ at an elevated electron temperature $\theta_{n}$ (Fig. 3). $V_{n}=V(1-n / N)$ is the potential in the $n$th cavity with $V=V_{L}-V_{R}$ the potential difference between left and right reservoir. The electron temperature $\theta_{n}$ follows from the energy balance equation using the WiedemannFranz law: ${ }^{12}$

$$
\theta_{n}^{2}=\theta^{2}+\frac{3 n(N-n)}{N^{2}}\left(\frac{e V}{\pi k_{B}}\right)^{2}
$$

Using Eqs. (4) and (5) the noise in this case of hot electrons is given by

$$
\begin{aligned}
S= & \frac{2}{N^{2}} \sum_{n=1}^{N}\left\{G k_{B}\left(\theta_{n}+\theta_{n-1}\right)\right. \\
& \left.+\sum_{k} \int_{0}^{\infty} d E T_{k n}\left(1-T_{k n}\right)\left[f_{n-1}-f_{n}\right]^{2}\right\} .
\end{aligned}
$$

The integral in Eq. (11) cannot be calculated analytically for the general case. Numerical results for the Fano factor are shown in Fig. 2(b). Analytical expressions can be given for $T_{i=1, \ldots, N}=1$, 


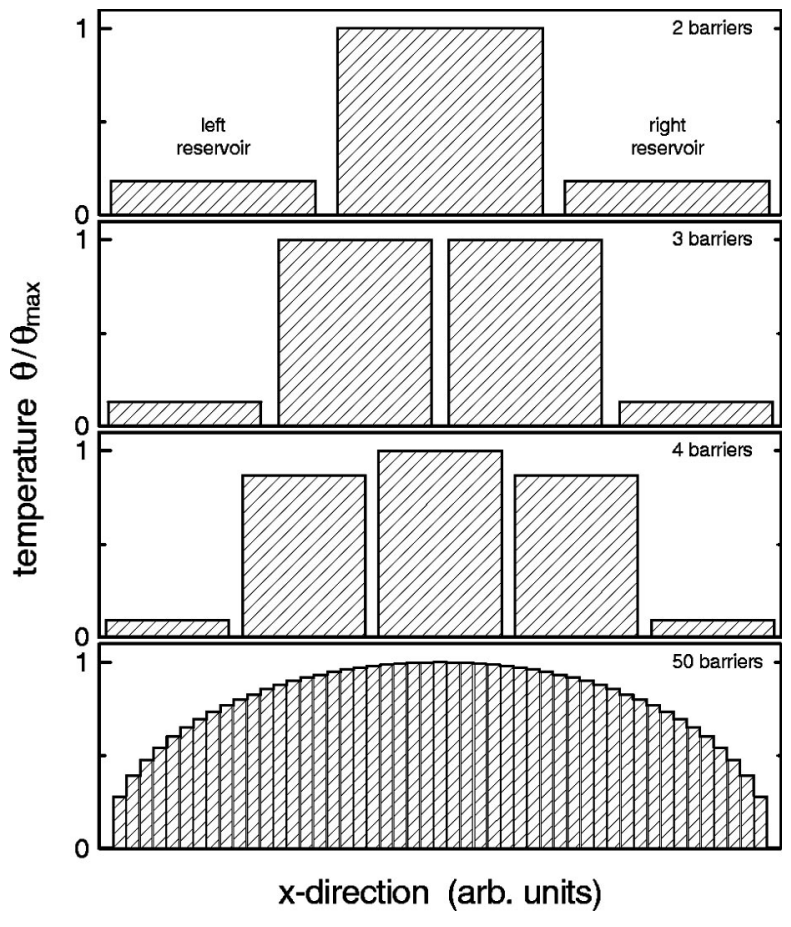

FIG. 3. Temperature profiles for 2, 3, 4, and 50 barriers in series. In case of inelastic electron-electron scattering electrons thermalize within the cavities and the temperature drastically increases above the bath temperature leading to enhanced noise. For a large number of barriers the temperature profile has a parabolic shape as for a metallic diffusive wire. ${ }^{13}$

$$
F=\frac{2 \sqrt{3}}{\pi N^{2}} \sum_{n=1}^{N-1} \sqrt{n(N-n)},
$$

and for the tunneling regime $\left(T_{i=1, \ldots, N} \ll 1\right)$ in the "diffusive limit" $(N \rightarrow \infty)$,

$$
F=\frac{2 \sqrt{3}}{\pi} \int_{0}^{1} d x \sqrt{x(1-x)}
$$

with $x=n / N \in[0,1]$. In both cases $F$ equals $\sqrt{3} / 4$ in the limit $N \rightarrow \infty$, in agreement with the result for a diffusive conductor with electron heating. ${ }^{14,15}$

\section{DEVICE}

Experimentally, a structure as described in Fig. 1 has been realized in a two-dimensional electron gas (2DEG) by a series of up to four QPC's across a wet-chemical etched Hall bar (Fig. 4). The 2DEG forms at the interface of a standard

$\mathrm{GaAs} / \mathrm{Al}_{0.3} \mathrm{Ga}_{0.7} \mathrm{As}$ heterojunction $80 \mathrm{~nm}$ below the surface. Its carrier density equals $2.7 \times 10^{15} \mathrm{~m}^{-2}$ and the mobility is $80 \mathrm{~V} \mathrm{~s} / \mathrm{m}^{2}$. The different split gates are spaced by $20 \mu \mathrm{m}$ and the Hall bar is $100 \mu \mathrm{m}$ wide. Thus, the size of the cavity is rather large so that thermalization of the electrons can take place inside the cavity. ${ }^{8}$

Voltage noise has been measured as a function of current for one to three QPC's in a series with fixed transmission probabilities. In the experiment, we used only up to three QPC's because one of the four did not show proper conduc-
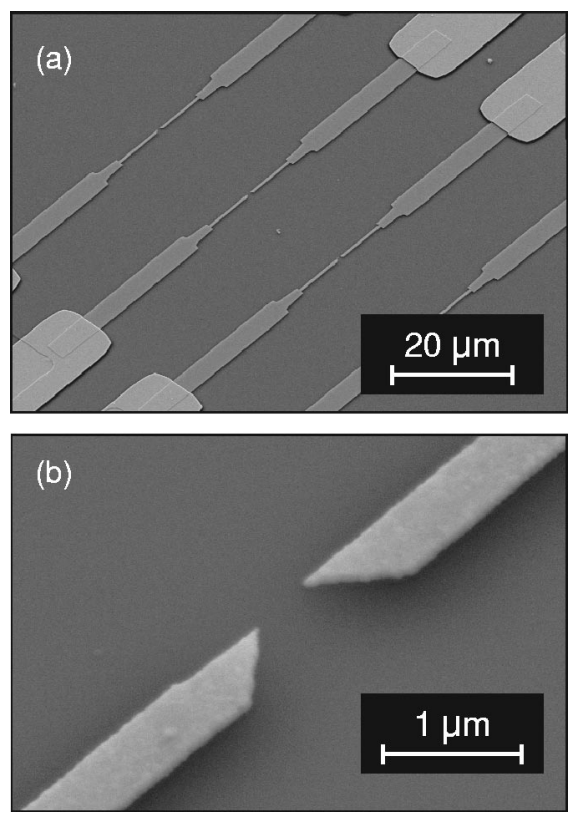

FIG. 4. Scanning electron microscope images of the measured device. (a) Four point contacts forming a sequence of large cavities. (b) The single point contacts are defined with split gates.

tion quantization. Since the gates do not influence each other, the transmission of a single point contact can be determined independently by measuring its conductance while the others are kept completely open. ${ }^{8}$ The spectral density of the voltage fluctuations are typically averaged over a frequency bandwith of $1 \mathrm{kHz}$ at around 7-9 kHz. Experimental details can be found in Refs. 5 and 8.

\section{RESULTS AND DISCUSSION}

In Fig. 5 the Fano factor $F \equiv S / 2 e|I|$ extracted from the shot-noise measurements is plotted as a function of the number of point contacts. The black dots correspond to experi-

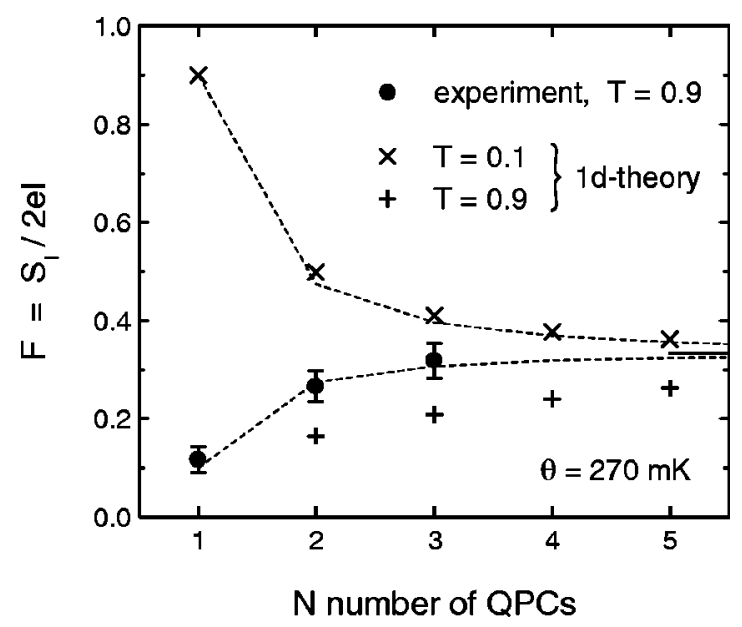

FIG. 5. The black points are experimental data for one mode transmitted at the point contacts with probability $T=0.9$. The dashed line are theoretical predictions including cavity noise for noninteracting electrons [Eq. (8)]. The crosses correspond to the one-dimensional model of de Jong and Beenakker. ${ }^{9}$ 
mental data obtained in the case that each single QPC is adjusted to a transmission probability $T \simeq 0.9$. The dashed lines are the prediction of Eq. (8) for $T=0.1$ (upper curve) and for $T=0.9$ (lower curve), whereas the crosses correspond to the one-dimensional calculation of Ref. 9. For $N$ $=1$ shot noise is strongly suppressed compared to $S_{\text {Poisson }}$ $=2 e|I|$, as expected for a single QPC with high transmission probability. ${ }^{3}$ When $N$ goes from 1 to 3 shot noise becomes larger. We find that the Fano factor increases faster with increasing number of the contacts $N$ than predicted by the onedimensional theory. For $N=3$ it is already very close to the $1 / 3$ value of the diffusive regime. The measured data are consistent with our theoretical prediction that takes the cavities into account. This demonstrates that in the system depicted in Fig. 1 not only partition noise at the contacts contributes to the total noise but also additional cavity noise. Since the cavities are large compared to the mean free path of the electrons the electrons stay relatively long in the cavity so that inelastical scattering should be present. However, the uncertainties of the experimental data due to small inequalities in the transparencies of the single QPC's and due to mode mixing do not allow to distinguish between the cold- and hot-electron regime in this experiment.

\section{CONCLUSIONS}

We studied the shot noise of a series of QPC's forming a sequence of cavities. Theoretical calculations along the lines of Ref. 7 show that the shot noise reaches $1 / 3$ and $\sqrt{3} / 4$ of the Poissonian limit for cold and hot electrons, respectively, when the number of point contacts $N$ is increased to infinity. Noise measurements on a series of 1,2, and 3 QPC's defined in a 2 DEG are in reasonable qualitative agreement with our calculation that takes the contribution from the cavities into account.

Discussions with B. Frei are kindly acknowledged.

\section{ACKNOWLEDGMENTS}

Discussions with B. Freiare kindly acknowledged. This work was supported by the Swiss National Science Foundation.
*Present address: Delft University of Technology, PO Box 5046, 2600 GA Delft, The Netherlands.

${ }^{1}$ For a recent review, see Ya.M. Blanter and M. Büttiker, Phys. Rep. 336, 1 (2000).

${ }^{2}$ G.B. Lesovik, JETP Lett. 49, 592 (1989); M. Büttiker, Phys. Rev. Lett. 65, 2901 (1990).

${ }^{3}$ A. Kumar et al., Phys. Rev. Lett. 76, 2778 (1996); M. Reznikov et al., ibid. 75, 3340 (1995).

${ }^{4}$ C.W.J. Beenakker and M. Büttiker, Phys. Rev. B 46, 1889 (1992).

${ }^{5}$ M. Henny, S. Oberholzer, C. Strunk, and C. Schönenberger, Phys. Rev. B 59, 2871 (1999).

${ }^{6}$ R.A. Jalabert, J.-L. Pichard and C.W.J. Beenakker, Europhys. Lett. 27, 255 (1994).

${ }^{7}$ Ya.M. Blanter and E.V. Sukhorukov, Phys. Rev. Lett. 84, 1280 (2000).

${ }^{8}$ S. Oberholzer et al., Phys. Rev. Lett. 86, 2114 (2001).

${ }^{9}$ M.J.M. de Jong and C.W.J. Beenakker, Phys. Rev. B 51, 16867 (1995).
${ }^{10}$ In a simple one-dimensional system, in which electrons tunnel through $N$ identical barriers, and where no correlations occur between current fluctuations at different barriers, the noise of all barriers is just averaged. The total voltage noise in this case would be $S_{V}=N\left(2 e|I| R^{2}\right)$ with $R$ the resistance of a single barrier. Consequently the Fano factor follows as $F$ $=S_{V} /(N R)^{2} / 2 e|I|=1 / N$ and the shot noise disappears with increasing $N$. This is the case, for example, in a series of $N$ vacuum tubes.

${ }^{11}$ A series of planar tunnel barriers can be modeled by series QPC's in the integer quantum Hall regime with no backsacttering in the cavities between the barriers.

${ }^{12}$ E.V. Sukhorukov and D. Loss, Phys. Rev. B 59, 13054 (1999).

${ }^{13}$ M. J. M. de Jong, Ph.D. thesis, Leiden University, 1995.

${ }^{14}$ K.E. Nagaev, Phys. Rev. B 52, 4740 (1995).

${ }^{15}$ A.H. Steinbach, J.M. Martinis, and M.H. Devoret, Phys. Rev. Lett. 76, 3806 (1996).

${ }^{16}$ S.A. van Langen and H. Büttiker, Phys. Rev. B 55, 1680 (1997). 